\title{
CODIUM PICTURATUM SP. NOV. (CHLOROPHYTA), UNA ESPECIE EXTRAORDINARIA DEL PACIFICO TROPICAL MEXICANO
}

\author{
Francisco F. Pedroche ${ }^{1}$ \\ Depto. de Hidrobiología, CBS \\ UAM-Iztapalapa \\ Apdo. Postal 55-535 \\ México, D.F. 09340, México \\ Y \\ Paul C. Silva \\ University Herbarium \\ University of California \\ Berkeley, CA 94720-2465, USA
}

\section{RESUMEN}

Se describe una nueva especie costrosa de Codium con un talo muy delgado, C. picturatum F.F. Pedroche et P.C. Silva, que crece desde la parte sur del Golfo de California a lo largo de la costa tropical del Pacífico mexicano, Panamá, Colombia y las Islas Galápagos. Anatómicamente es muy diferente del resto de las especies costrosas, incluyendo $C$. hubbsii Dawson y C. setchelli Gardner, dos miembros pertenecientes al grupo de $C$. adhaerens conocidos con anterioridad para el Pacífico de México. En estas especies, utrículos hijos se producen por gemación de los utrículos parentales y forman agrupamientos que son fáciles de separar por disección. En C. picturatum, los utrículos hijos se forman en el extremo de filamentos producidos por los parentales, como ocurre en las especies de talos erectos y ramificados, pero la mayoría de los filamentos se encuentran acortados a tal punto que los utrículos hijos nacen directamente sobre el que los origina. Los filamentos medulares son tortuosos y ramificados cercanamente, lo que dificulta la disección del talo.

\begin{abstract}
A very thin crustose Codium, occurring in the southern part of the Gulf of California and along the tropical Pacific coast of Mexico southward to Panama, Colombia, and the Galapagos Islands, is described as a new species, C. picturatum F.F. Pedroche et P.C. Silva. Its anatomy is very different from that of other crustose species, including $C$. hubbsii Dawson and $C$. setchellii Gardner, two members of the $C$. adhaerens group known from Pacific Mexico. In that group, daughter utricles are produced by budding from parent utricles, resulting in clusters that are easily separated by dissection. In C. picturatum daughter utricles are formed at the end of filaments produced by parent utricles, as in erect, branched thalli, but with most filaments being shortened, some to the point that daughter utricles are borne directly on the parent utricle. The medullary filaments are tortuous and closely branched, so that dissection of the thallus is difficult.
\end{abstract}

${ }^{1}$ Dirección actual: University Herbarium, University of California, Berkeley, CA 94720-2465, USA. 
Los litorales de México son bien conocidos por sus diversas e interesantes ficofloras marinas. Las costas del Atlántico y Caribe comparten la flora tropical que se distribuye a ambos lados del ecuador, de Brasil a Bermuda, y aunque la representación algal en nuestro país es rica en especies, incluye pocas si es que ninguna, completamente exclusiva en México. En contraste, la costa del Pacífico comprende tres áreas florísticas: Baja California Occidental incluyendo las islas oceánicas, de las cuales destaca Isla Guadalupe; el Golfo de California; y el Pacífico mexicano al sur de Mazatlán. Las dos primeras regiones resaltan por su alto grado de endemismo, y mientras que la tercera es relativamente pobre en especies, muestreos intensivos están demostrando la existencia de algas, con distribución limitada, no descritas anteriormente. Uno de esos taxa es el que se describe a continuación.

En 1978 se recolectó un talo costroso muy delgado, perteneciente al género Codium, en la región de Chamela, Jalisco (Pedroche y González González, 1981, como Codium sp.). Este fragmento carecía de estructuras reproductoras, pero pesquisas subsecuentes en localidades desde Playa La Audiencia, Colima hasta Isla La Roqueta en Guerrero, hacia el sur, brindaron material fértil. Tales colecciones integraron un número suficiente de poblaciones como para permitir la caracterización completa de esta especie tan singular. Durante el desarrollo del estudio, la distribución conocida de $C$. picturatum se ha extendido hacia el norte, a la parte sur del Golfo de California, y hacia el sur a la costa de Panamá, Colombia y las Islas Galápagos.

Todas las muestras fueron fijadas en formol neutro a $4 \%$ preparado con agua de mar. Los dibujos se efectuaron con la ayuda de una cámara clara.

Codium picturatum F. F. Pedroche et P. C. Silva, sp. nov. (Figs. 1-6).

Thallus applanatus, 1-2 mm crassus, firme spongiosus, sustrato firme adhaerens, crustam irregularem indefinatamque sine marginibus libris formans, pagina laevi. Utriculi difficulter disparandi, greges parvas sed non familias utriculorum formantes. Utriculi primarii ex illis secundariis non distinguibiles, uterque ex filamentis orientibus irregularissime e parte basali utriculorum vel e filamentis medullosis sympodialiter formati, obturaculium in loco originis e filamento evolutum.

Utriculi subcylindrici vel plusminusve claviformes, (270-) 400-900 (-1200) $\mu \mathrm{m}$ long., (38-) 55-85 (-125) $\mu \mathrm{m}$ diam. Apices rotundati, membrana utricularis tenuis $(1.5 \mu \mathrm{m})$, ad apicem crassescens $(-2.4 \mu \mathrm{m})$. Pili et cicatrices aliquando adsunt, 1-aliquot per utriculo, (45-) 60-115 (-160) $\mu \mathrm{m}$ infra apicem portati. Filamenta medullosa tortuosa et arcte ramificata, maximam partem 20-35 $\mu \mathrm{m}$ diam., plerumque haptera formantia, stratum tenue infra vallum utricularem efferentia. Gametangia fusiformia, 160-240 (-270) $\mu \mathrm{m}$ long., 48-

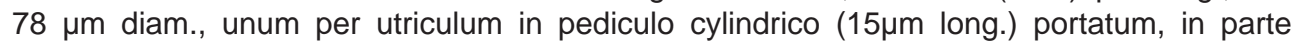
(190-) 230-335 (-365) $\mu \mathrm{m}$ infra apicem inventa.

Talo aplanado de 1-2 mm de grosor, superficie lisa, textura firme-esponjosa, firmemente adherido al substrato formando una costra irregular e indefinida sin márgenes libres.

Disección del talo difícil, resultando en grupos pequeños de utrículos, pero sin formar familias. Utrículos primarios indistinguibles de los secundarios, todos formados simpodialmente a partir de filamentos que surgen, de manera altamente irregular, de la región basal de 
Pedroche y Silva: Codium picturatum, Una Especie Extraordinaria del Pacífico Tropical Mexicano

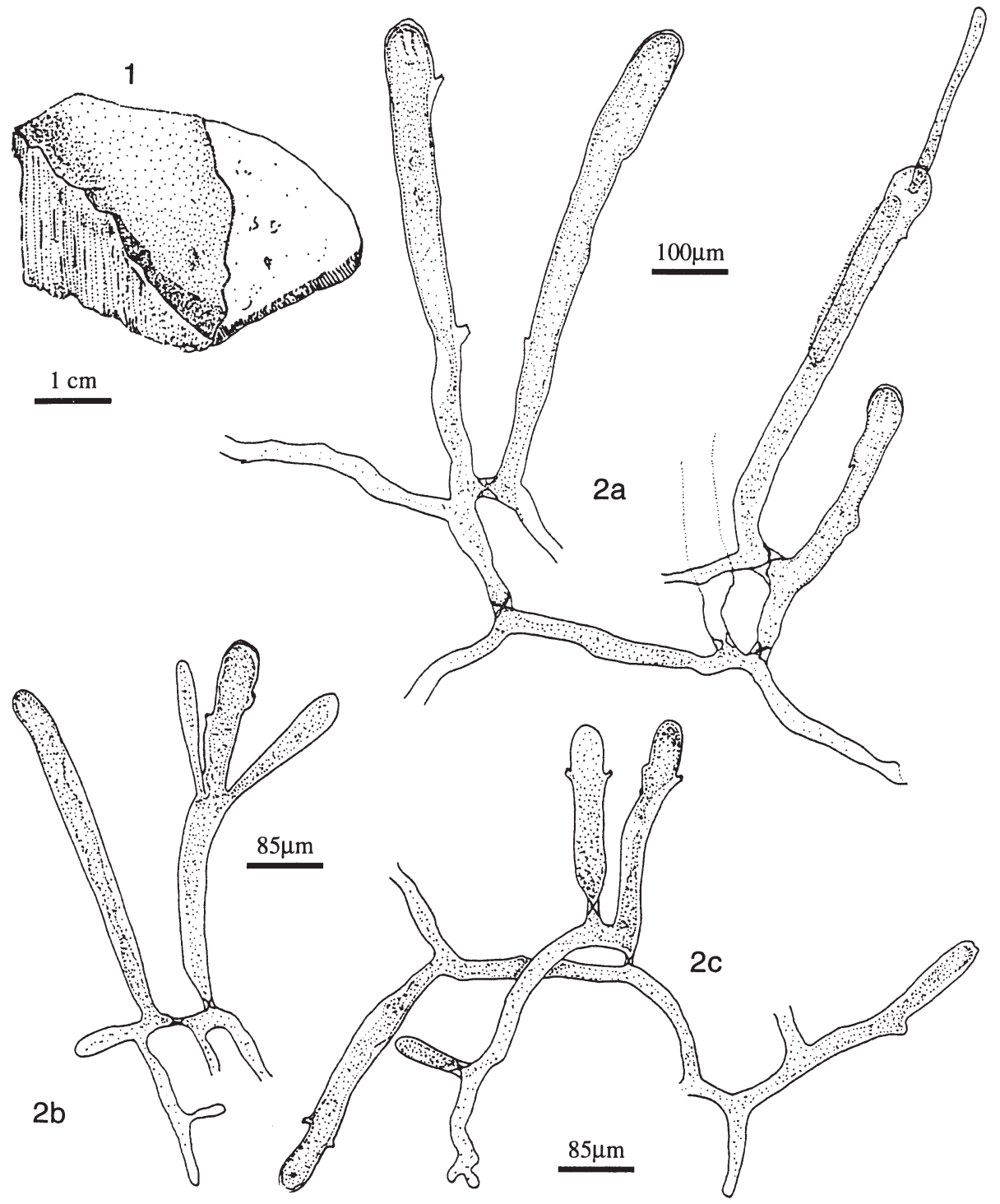

Figs. 1 y 2. Codium picturatum. 1. Hábito sobre una roca (Playa La Audiencia, Col.). 2. Diversidad de utrículos: a: Mezcález, Jal.; b y c: Zihuatanejo, Gro.

utrículos ya existentes o de otros filamentos medulares, con un septo desarrollándose en el punto de origen del filamento. Utrículos subcilíndricos a ligeramente claviformes (270-) 400-900 (-1200) $\mu \mathrm{m}$ en longitud, (38-) 55-85 (-125) $\mu \mathrm{m}$ en diámetro. Apices redondeados, 
pared utricular delgada $(1.6 \mu \mathrm{m})$, ligeramente engrosada $(-24 \mu \mathrm{m})$ en los ápices. Pelos o cicatrices ocasionales, de uno a varios por utrículo, situados entre (45-) 60-115 (-160) $\mu \mathrm{m}$ del ápice. Filamentos medulares tortuosos y ramificados cercanamente, la mayoría de 20-35 $\mu \mathrm{m}$ de diámetro, comúnmente formando estructuras de fijación, integrando una capa fina por debajo de la palizada de utrículos.

Gametangios fusiformes, 160-240 (-270) $\mu \mathrm{m}$ en longitud, 48-78 $\mu \mathrm{m}$ de diámetro, uno por utrículo naciendo sobre un pedicelo cilíndrico de $15 \mu \mathrm{m}$ de longitud, situados en una zona entre (190-) 230-335 (-365) $\mu \mathrm{m}$ por debajo del ápice.

Holotipo: Playa La Audiencia [cerca Manzanillo], Colima, México. Sobre rocas expuestas en marea baja, en áreas de oleaje moderado. 22.III.1991, Pedroche et al. FP12ne (MEXU, isotipos en UAMIZ y UC).

Nombre mal aplicado: Codium setchellii. Taylor, 1945, pág. 68 (pro parte); Riosmena Rodríguez et al., 1992, pág. 14; León et al., 1993, pág. 199. El registro de Brusca y Thomson (1975) para Bahía Pulmo, en la parte sur del Golfo de California, se refiere seguramente a $C$. picturatum, aunque no hemos tenido oportunidad de revisar los ejemplares.

Codium picturatum es también la especie mencionada por Dawson (1959, pág. 16) como "a thin, prostrate species" del Golfo de California; así mismo, Codium spec. de Schnetter y Bula Meyer (1982, págs. 39-40) para Colombia.

Material adicional: MEXICO. Baja California Sur. Isla San Ildefonso, 24.IV.1958, E. Y. Dawson 18725 (LAM); Punta Perico, Isla Carmen, 30.III.1982, P. C. Silva y R. L. Moe 7-24 (UC); Puerto Escondido, 22.IV.1958, E. Y. Dawson 18779 (UC, LAM); Bahía Agua Verde, 20.IV.1958, E. Y. Dawson 18911 (LAM); Punta San Evaristo, 19.IV.1958, E. Y. Dawson 18956 (LAM); lado este de Isla Partida, 28.IV.1958, E. Y. Dawson 18587 (LAM); entre Isla Espíritu Santo e Isla Partida, 17.IV.1958, E. Y. Dawson 18932 (UC, LAM); La Lobera, Isla Espíritu Santo, 17.V.1991, Neri Melo s.n. (FBCS); Calerita, 8.V.1990, Riosmena Rodríguez s.n. (FBCS). Jalisco. Chamela, 3.IV.1978, Pedroche et al. ch-2-36 (FCME); Mezcález, 21.III.1991, Pedroche et al. FP5ne (UAMIZ); Tenacatita, 21.III.1991, Pedroche FP28ne (UAMIZ). Michoacán. Caleta de Campos, 1.IX.1990, D. Fragoso y D. León PTM 3964 (FCME). Guerrero. Las Gatas, Zihuatanejo, 24.VI.1981, Pedroche s.n. (UAMIZ); Isla Roqueta, Acapulco, 29.III.1984, Candelaria s.n. (FCME). PANAMA. I. Jicarita, 21.II.1934 W. R. Taylor 34-508 (UC). COLOMBIA. Isla Gorgonilla, 21.V.1979, G. Bula C-186 (Herb. Bula Meyer). ECUADOR. Islas Galápagos. Isla Pinta, 2.II.1933, Schmitt 46A-33 (MICH); Isla Santa María, W. R. Taylor 34-242 (LAM, MICH).

Habitat: Crece adherido a rocas y conchas de bivalvos, libres de arena, en zona mesomareal baja o inframareal somera.

Distribución conocida (Fig. 6): Golfo de California $\left(26^{\circ} 37^{\prime} N\right)$, México a las Islas Galápagos, Ecuador (1¹5’S).

El epíteto de esta especie proviene del latin picturatus, palabra que significa pintado y que alude a la apariencia de este talo muy delgado, sobre el sustrato. 
Pedroche y Silva: Codium picturatum, Una Especie Extraordinaria del Pacífico Tropical Mexicano

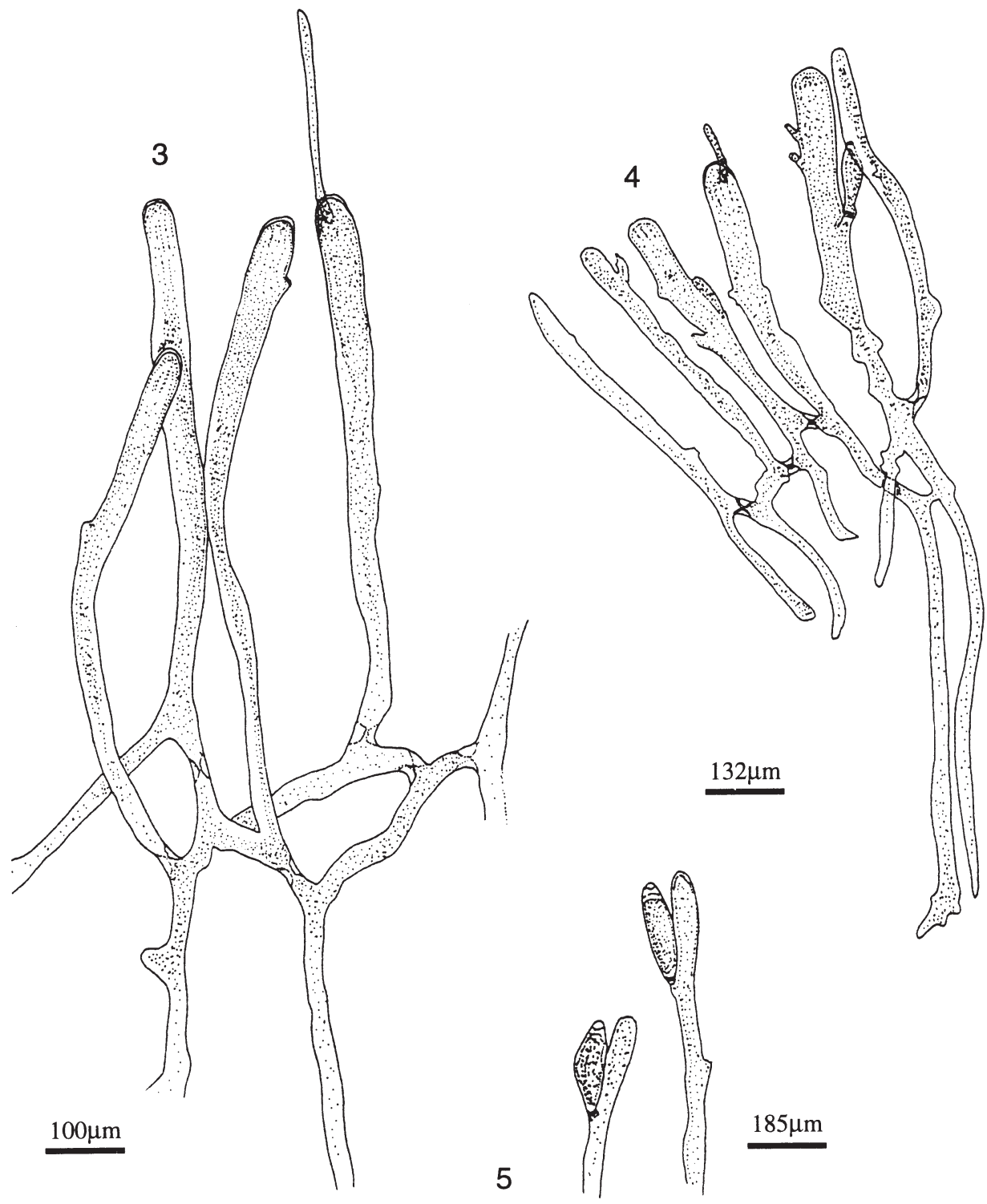

Figs. 3-5. Codium picturatum. 3. Utrículos de la porción central del talo, mostrando el septo en la base y pelo apical (Mezcález, Jal.). 4. Secuencia de utrículos en los que se observa la reducción del crecimiento simpodial y los procesos de fijación en algunos filamentos medulares (Playa La Audiencia, Col.). 5. Gametangios en la porción superior de los utrículos (Ibid.). 


\section{DISCUSION}

En el título que decidimos utilizar, el adjetivo "extraordinaria" se refiere a las características anatómicas, casi únicas para éste género. Mientras que el talo tiene, a simple vista, parecido con otras especies costrosas (por ejemplo, C. hubbsii Dawson y C. setchelli Gardner en la flora ficológica del Pacífico de Baja California), el desarrollo y la estructura madura de los utrículos difieren completamente de lo observado en esos taxa. Codium hubbsii se encuentra principalmente en las islas de California del Sur y de Baja California, desde Isla Santa Catalina hasta Rocas Alijos. A lo largo de la costa peninsular, ha sido hallada en Punta Malarrimo y Bahía Asunción solamente (Pedroche y Silva, en prep.). Codium setchellii se distribuye desde Sitka, Alaska, hasta Punta Abreojos, Baja California, pero está ausente en las islas mexicanas (Pedroche y Silva, en prep.).

Codium hubbsii y $C$. setchellii son miembros del grupo $C$. adhaerens, en el cual utrículos hijos se producen por gemación de los utrículos parentales, dando por resultado agrupaciones (familias) fácilmente disgregables por disección, carácter por mucho tiempo empleado para distinguir al subgénero Tylecodium (Setchell en Lucas, 1935).

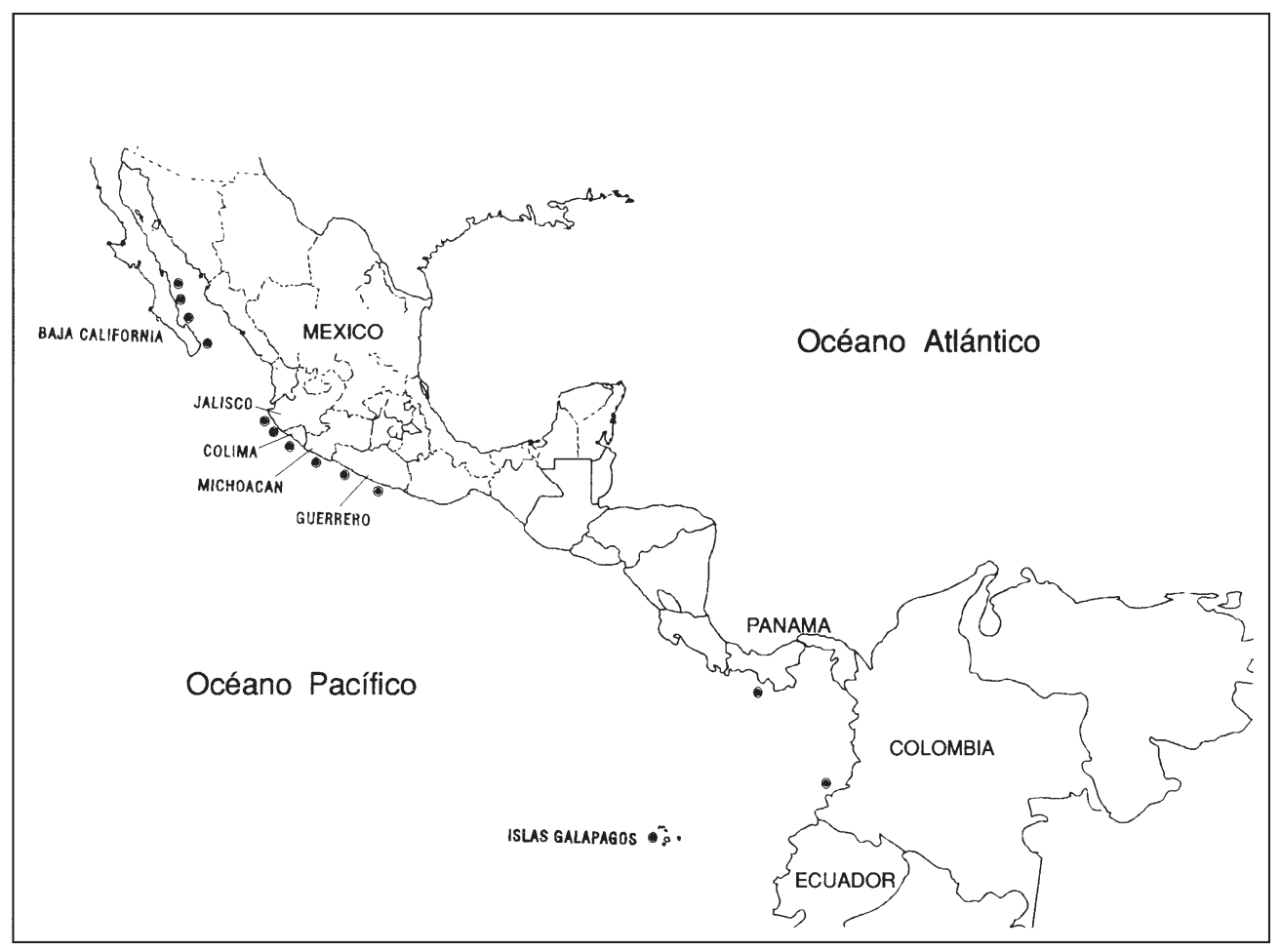

Fig. 6. Codium picturatum. Distribución conocida. 
En C. picturatum, todos los utrículos se desarrollan, aparentemente, a partir de los ápices curvados de filamentos simpodiales, como en las especies erectas y dicotómicamente ramificadas. Sin embargo, la formación utricular en $C$. picturatum es altamente irregular, con la mayoría de los filamentos acortados, algunos al extremo de que un nuevo utrículo nace directamente en el utrículo existente. En esta última situación, un septo, que normalmente se desarrolla en el punto de origen del filamento simpodial, aparece en la base de un nuevo utrículo (Fig. 2). La palizada de utrículos está sostenida por una capa muy delgada de filamentos medulares, los cuales son tortuosos, con frecuencia ramificados cercanamente, y firmemente adheridos al sustrato, haciendo difícil la disección del talo.

El desarrollo de un septo en la base de la mayoría de los utrículos ofrece un parecido superficial con C. pelliculare de Sudáfrica (Silva, 1959). Sin embargo, el talo de esta especie es membraniforme y cuando madura es removido facilmente del sustrato. También, la formación de los utrículos es mucho más ordenada que en $C$. picturatum. Desde el punto de vista anatómico, la especie más cercanamente relacionada parece ser $C$. giraffa Silva (1979), a pesar de su hábito erecto. Esta alga, que tiene una distribución similar (Nayarit a Oaxaca), está restringida a situaciones mesomareales con fuerte exposición al oleaje pero también muestra una formación irregular de utrículos, involucrando en su mayoría, filamentos simpodiales reducidos.

Actualmente nos encontramos realizando la monografía del género Codium para el Pacífico mexicano y los resultados comparativos de morfología, anatomía, genética molecular y distribución, auxiliados por las técnicas modernas en cladística, podrán brindarnos una idea de las relaciones filogenéticas entre las diversas especies presentes a lo largo del mencionado litoral.

\section{AGRADECIMIENTOS}

Francisco F. Pedroche agradece a la Organización de Estados Americanos (OEA) por la ayuda recibida, a través del programa PRA, para la realización del presente estudio. Agradecemos la cooperación de Michael Wynne al proporcionarnos los ejemplares depositados en $\mathrm{MICH}$, a Rafael Riosmena por las muestras procedentes de FBCS, así como a Don Reynolds, curador de LAM. De mucha ayuda fueron los comentarios de Max Chacana y de Gabriela Parra al manuscrito original; agradecemos asimismo el trabajo de Richard L. Moe en la diagnosis latina.

\section{LITERATURA CITADA}

Brusca, R. C. y D. A. Thomson. 1975. Pulmo reef: the only "coral reef" in the Gulf of California. Ciencias Marinas 2: 37-53.

Dawson, E. Y. 1959. Marine algae from the 1958 cruise of the Stella Polaris in the Gulf of California. Los Angeles County Museum Contributions in Science 27. 39 pp.

León, H., D. Fragoso, D. León, C. Candelaria, E. Serviere y J. González González. 1993. Characterization of tidal pool algae in the Mexican tropical Pacific coast. Hydrobiologia 260/261: 197-205.

Lucas, A. H. S. 1935. The marine algae of Lord Howe Island. Proceedings of the Linnean Society of New South Wales 60: 194-232. 
Pedroche, F. F. y J. González González. 1981. Lista florística preliminar de las algas marinas de la región sur de la costa de Jalisco, México. Phycologia Latino-Americana 1: 60-71.

Riosmena Rodríguez, R., D. A. Siqueiros Beltrones, O. García de la Rosa y V. Rocha Ramírez. 1992. ("1991"). The extension geographic range of selected seaweeds on the Baja California peninsula. Revista de Investigación Científica 2(2): 13-20.

Schnetter, R. y G. Bula Meyer. 1982. Marine Algen der Pazifikküste von Kolumbien. Chlorophyceae, Phaeophyceae, Rhodophyceae. Bibliotheca Phycologica 60. 287 pp.

Silva, P. C. 1959. The genus Codium (Chlorophyta) in South Africa. Journal of South African Botany 25: 103-165.

Silva, P. C. 1979. Codium giraffa, a new marine green alga from tropical Pacific Mexico. Phycologia 18: 265-268.

Taylor, W. R. 1945. Pacific marine algae of the Allan Hancock Expeditions to the Galapagos Islands. Allan Hancock Pacific Expenditions 12. 528 pp. 